the darwinian view of evolution as a progress toward adaptation and fitness.

Miller died three years before Darwin's Origin of Species was published. He is, as Taylor puts it, "regarded as a loser in the crucial evolutionary debate... That is simply because it never really began in Miller's lifetime." But Miller, along with other contemporary palaeontologists, paved the way to evolutionary concepts. All that was missing was a process that did not need divine intervention, and Darwin provided it.

Hugh Miller is superbly written, clear and readily accessible to those who have no background in geology, palaeontology or Scottish history. It is to be strongly recommended to historians of science, lay naturalists and any reader interested in Scottish life and history.

Philippe Janvier is at the CNRS, Département

Histoire de la Terre, Muséum National d'Histoire Naturelle, 75005 Paris, France.

\title{
Brain botch
}

\section{The Accidental Mind: How Brain Evolution Has Given Us Love, Memory, Dreams, and God \\ By David Linden \\ Harvard University Press: 2007. 288 pp. \\ $\$ 25.95, £ 16.95$}

\section{Georg Striedter}

The human brain, and hence the human mind, is not an optimal, designed-fromscratch apparatus. Rather, it is an imperfect amalgam of shoddy components. That is the central thesis of David Linden's new book The Accidental Mind. Neurons are slow, leaky, and unreliable - hardly ideal computing elements. The whole brain, too, is not designed to the plan of some omnipotent engineer. Instead, evolution has endowed it with plenty of 'anachronistic junk'. Which is why, according to Linden, our minds often distort reality and can lead us to act foolishly. For example, when you reach out to touch something, your brain filters out what it expects. This selective neglect of expected input allows us to focus on unexpected stimuli, but it can be counterproductive. It may explain, for instance, why pushing and shoving confrontations tend to escalate. When someone pushes you, you feel it more than when you push the other with the same force, because the sensation caused by your own push is largely, though unconsciously, expected by your brain.

Linden tells his story well, in an engaging style, with plenty of erudition and a refreshing honesty about how much remains unknown. The book should easily hold the attention of readers with little background in biology and no prior knowledge of brains. It would make an excellent present for curious non-scientists and a good book for undergraduates who are just entering into the brain's magic menagerie. Even readers trained in neuroscience are likely to enjoy the many tidbits of rarely taught information - on love, sex, gender, sleep and dreams - that spice up Linden's main argument. The Accidental Mind stands out for being highly readable and clearly educational. No doubt, the human brain evolved along a constrained path and is, in some respects, designed imperfectly. Linden will send that message home.

Regrettably, Linden neglects to cover some material that could have boosted his thesis.

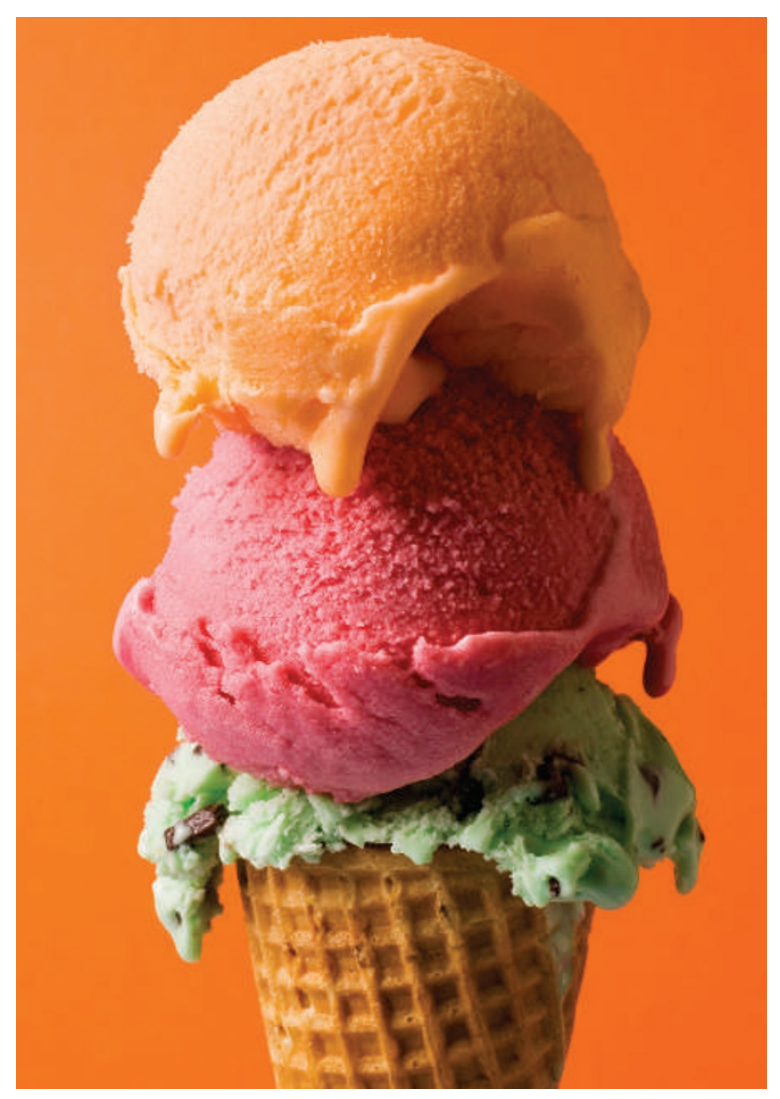

Brain evolution: new scoops on an old cone?

Particularly interesting would have been a discussion of the various "fast and frugal heuristics" that humans use to understand the world (for example, if you recognize one object but not another, then the former is probably bigger, better or more valuable). Even though such heuristics may sometimes yield inaccurate results, they evolved because they are generally 'good enough' and faster to execute than 'optimal' cognitive strategies. This, incidentally, is why such heuristics are used by engineers to build autonomous robots. Old-style robots that try to analyse their world veridically by computing all costs and benefits of possible actions, were slow, fragile and cumbersome. The newer robots act foolishly in some contexts, but they are fast and effective in their normal terrain. In many ways, they imitate our brains.

Another area Linden oddly neglects is evolutionary neuroscience. This field has made impressive strides in the past 20 years, but instead of discussing these, Linden reiterates the now outdated theory that mammal brains evolved by adding a neocortex to a "reptilian brain core". This theory is probably false, as most experts agree that the mammalian neocortex evolved out of a structure that exists in all reptiles, though the reptilian cortex does not have the complexity or size of its mammalian counterpart. Amending Linden's analogy, one might say that human brains evolved not by the addition of new scoops to an old ice cream cone, but by the modification of pre-existing scoops. This insight would actually have bolstered Linden's thesis that brains are subject to historical constraints. More difficult to show is that the use of pre-existing parts imposes functional constraints or 'bad design'.

Linden does write about some functional constraints on human brains, such as neuronal noise. This is an interesting idea, but noisy neurons may be flawed mainly in comparison to standard computer components. A shift in perspective suggests that noisy neurons, assembled en masse, excel at overcoming component failure (that is, brain lesions). Indeed, in the rough and tumble world of real organisms, fault-tolerance may well be more vital than ultrafast, exhaustive computing. In other words, in order to distinguish neuronal design features from bugs, we need to know the brain's performance specifications, which still remain debatable. One could reasonably argue, for example, that pushing your opponent harder than they pushed you is adaptive, or good design in evolutionary terms, because it demonstrates your physical combat strength efficiently.

Linden is right to stress that brains evolved, but hasty to conclude that they are flawed in their design. We still know too little about the brain's inner workings to judge how well it does its job. What we do know, and what The Accidental Mind helps us to realize, is that the human brain is not designed as many have imagined. Our brains are not hydraulic devices (as Descartes had claimed), phone switchboards or desktop computers. All those analogies are weak. Indeed, our predilection for solving problems by analogy often misleads. Still, analogical thinking probably worked well enough in our past to be selected for. Whether we view it as a boon or a bug depends on our perspective.

Georg Striedter is an associate professor at the

Department of Neurobiology and Behaviour, Univ. of California, Irvine, California 92697, USA. 